\title{
NRG1-dependent activation of HER3 induces primary resistance to trastuzumab in HER2-overexpressing breast cancer cells
}

\author{
LIUTING YANG ${ }^{1,2}$, YINGYING LI ${ }^{3}$, ENYUN SHEN $^{4}, \mathrm{FENGQI} \mathrm{CAO}^{4}, \mathrm{LI} \mathrm{LI}^{2}, \mathrm{XIAOJIN} \mathrm{LI}^{5}$, \\ XUEJIANG WANG ${ }^{3}$, SEYED KARIMINIA ${ }^{6}$, BINGMEI CHANG ${ }^{1}$, HONGZHONG LI $^{7}$ and QIN LI $^{2}$ \\ ${ }^{1}$ Department of Biochemistry and Molecular Biology, Basic Medical College, Shanxi Medical University, \\ Taiyuan, Shanxi 030001; ${ }^{2}$ Department of Oncology, Beijing Friendship Hospital, Capital Medical University, \\ Beijing 100050; ${ }^{3}$ Department of Pathology and Pathophysiology, Basic Medical College, Capital Medical University, \\ Beijing 100054; ${ }^{4}$ Beijing Cotimes Biotech Co., Ltd., Beijing 100176; ${ }^{5}$ Experimental Center, Beijing Friendship Hospital, \\ Capital Medical University, Beijing 100050, P.R. China; ${ }^{6}$ Molecular and Cellular Oncology, MD Anderson \\ Cancer Center, Houston, TX 77030, USA; ${ }^{7}$ Chongqing Key Laboratory of Molecular Oncology and Epigenetics, \\ The First Affiliated Hospital of Chongqing Medical University, Chongqing 400416, P.R. China
}

Received May 13, 2017; Accepted September 15, 2017

DOI: $10.3892 /$ ijo.2017.4130

\begin{abstract}
This study was conducted to determine the role of neuregulin 1 (NRG1)-dependent human epidermal growth factor receptor 3 (HER3) activation in trastuzumab primary resistance, and to observe the inhibitory effect of HER3 monoclonal antibody on HER2-overexpressing breast cancer cells. BT474 cells (trastuzumab sensitive) and MDA-MB-453 cells (trastuzumab resistant) were first stimulated with NRG1 and then treated with either trastuzumab, HER3 antibody, or a combination of both. The expression of phospho human epidermal growth factor receptor 2 (p-HER2), phospho human epidermal growth factor receptor 3 (p-HER3), phospho protein kinase $\mathrm{B}(\mathrm{p}-\mathrm{Akt}$ ) and phospho mitogen-activated protein kinase (p-MAPK) were detected by western blotting. Apoptosis was detected by flow cytometry. Cell viability was detected by
\end{abstract}

Correspondence to: Dr Qin Li, Department of Oncology, Beijing Friendship Hospital, Capital Medical University, 95 Yongan Road, Xicheng, Beijing 100050, P.R. China

E-mail: oncologistinbj@163.com

Abbreviations: HER, human epidermal growth factor receptor; p-HER2, phospho human epidermal growth factor receptor 2; p-HER3, phospho human epidermal growth factor receptor 3; p-Akt, phospho protein kinase B; p-MAPK, phospho mitogen-activated protein kinase; HER1, human epidermal growth factor receptor 1; HER2, human epidermal growth factor receptor 2; HER3, human epidermal growth factor receptor 3; HER4, human epidermal growth factor receptor 4; Akt, protein kinase B; MAPK, mitogen-activated protein kinase; NRG1, neuregulin 1; PI3K, phosphatidylinositol 3 kinase; siRNA, small interfering RNA; FBS, fetal bovine serum

Key words: breast cancer, trastuzumab, HER3 monoclonal antibody, HER 2, HER3
MTT assay. Without NRG1 stimulation, trastuzumab treatment significantly downregulated the expression of p-HER2, increased early apoptosis, and decreased cell viability in BT474 cells. After NRG1 stimulation, the aforementioned effects weakened or disappeared in the trastuzumab treatment group, whereas in the HER3 antibody treatment group, there was significant downregulation in p-HER3 expression and increase in early apoptosis of BT474 cells. In MDA-MB453 cells, the HER3 antibody significantly downregulated both p-HER 2 and p-HER 3 and promoted early apoptosis after NRG1 stimulation, however, trastuzumab hardly played a role. p-Akt and p-MAPK were also significantly downregulated by the HER3 antibody after NRG1 stimulation. The expressions of p-HER2, p-HER3, p-Akt and p-MAPK were all downregulated after HER3 gene silencing, compared to the control. NRG1-dependent activation of HER3 induces primary resistance to trastuzumab in HER2-overexpressing breast cancer cells. HER3 monoclonal antibody combined with trastuzumab may serve as a treatment choice for patients with primary resistance to trastuzumab.

\section{Introduction}

Human epidermal growth factor receptor (HER2)overexpressing breast cancer accounts for $20-30 \%$ of the total number of breast cancer (1), and the HER2 expression level is as much as 100 times higher when compared to non-cancerous breast tissue (2). HER2-overexpressing breast cancer has a higher degree of malignancy and is more prone to drug resistance than luminal A and luminal B, thus resulting in poorer prognosis and shorter survival time (3). Trastuzumab (Herceptin) is the first targeted anti-cancer drug in breast cancer, and it brings a remarkable breakthrough in the prognosis of HER2-overexpressing breast cancer (4). After binding to the HER2 receptor, trastuzumab inhibits the formation of HER2 heterodimers and the activation of downstream signaling pathways, which inhibits the proliferation and 
metastasis of breast cancer cells. Studies reported that patients with relapsed metastatic breast cancer have a median overall survival of up to 37.4 months and a median progression-free survival of up to 13.6 months after receiving trastuzumab combined with chemotherapy $(5,6)$.

Although trastuzumab has brought significant clinical benefits for breast cancer patients, primary and secondary resistance to trastuzumab has caused great confusion for clinicians and patients. The molecular mechanisms of trastuzumab resistance are poorly understood. It may be related to many factors. One factor is the absence of an effective binding site for HER2 or the destruction of molecular structure of HER2. Other possible factors include the reactivation of HER2related or unrelated signaling pathways $(7,8)$, changes in receptor function induced by the rearrangement of HER dimer axis (9-11), the abnormal activation of the phosphatidylinositol 3 kinase/protein kinase B (PI3K/Akt) signaling pathway, or the loss of phosphatase and tensin homolog deleted on chromosome ten (12-14).

HER3 is one of the members of HER family. The HER3 kinase domain cannot catalytically activate other kinases, due to lack of crucial residues, was once considered as a signaling substrate for other HER members. Therefore, the clinical value and research of HER3 were greatly ignored in the past. Currently, researchers found that its kinase domain possesses a specific allosteric activation function and acts as an activator kinase to activate receptor kinases (HER1, HER2, HER4) by forming heterodimers (15). Another study revealed HER3 could automatically phosphorylate since it has the capability to bind ATP by using its intracellular segments (16). HER3 has been found to be involved in the tumorigenesis and progression in many kinds of cancer. A study by Lee-Hoeflich et al demonstrated that HER3 formed powerful carcinogenic dimers with HER2, and that HER 3 was as important as HER 2 in promoting proliferation of HER2-overexpressing breast cancer cells (17). The cooperation between HER 2 and HER 3 is unique, but the underlying molecular mechanism still needs further study.

NRG1 acts as a ligand of HER3 and promotes the interaction of HER2/HER3 (18). NRG1/HER3 has been confirmed to promote cell proliferation through autocrine or paracrine modes in ovarian and colon cancer cells $(19,20)$. There is less research on the effects of NRG1/HER3 of breast cancer cells. It has been found that NRG1 upregulated the expression of matrix metalloproteinase-1/matrix metalloproteinase- 9 and promoted invasion of HER2-overexpressing breast cancer cells. One study showed that HER3 gene silencing downregulated NRG1 level and inhibited tumor cell invasion, and in turn, NRG1 gene silencing inhibited HER3 activity and cell proliferation (21). This study indicated that HER3 promoted the proliferation of HER2-overexpressing breast cancer cells in an NRG1-dependent manner. Moreover, a study by Göstring et al demonstrated that the activation loop of NRG1/HER3, independent of HER2, played a vital role in the proliferation process of HER2-overexpressing breast cancer cells (22).

In light of the promoted role NRG1/HER3 has in the proliferation of HER2-overexpressing breast cancer cells, the inactivation of NRG1-dependent HER3 signal path must play an important role in the inhibition of tumor cell growth when trastuzumab downregulates HER2. Otherwise, primary resistance to trastuzumab would occur. The study was performed to determine the role of NRG1-dependent HER3 activation in primary resistance to trastuzumab, and to clarify the inhibitory effect of HER3 monoclonal antibody on HER2-overexpressing breast cancer cells. HER2 and HER3 were both highly expressed in MDA-MB-453 and BT474 cells. One reference showed that MDA-MB-453 belongs to HER2enriched subtype based on the PAM50 gene signature (23), and it was also shown as intrinsic trastuzumab-resistant, HER2-positive breast cancer cell line $(24,25)$. Thus, we chose BT474 and MDA-MB-453 cell lines as our research objective.

\section{Materials and methods}

Cell culture, reagents and antibodies. BT474 and MDA-MB453 cell lines were purchased from the Institute of Basic Medical Sciences, Chinese Academy of Medical Sciences. BT474 cells (26) were cultured in DMEM/high-glucose medium. MDA-MB-453 cells (11) were cultured first in L-15 Leibovitz medium and then in modified RPMI medium. $1 \%$ penicillin-streptomycin and 20\% FBS were added to all media. The cells were cultured in a petri dish at $37^{\circ} \mathrm{C}$ and in a humidified atmosphere containing $5 \% \mathrm{CO}_{2}$. After incubation without FBS for $4 \mathrm{~h}$, the cells were treated with anti-human HER2 antibody trastuzumab (Herceptin) $(54 \mu \mathrm{g} / \mathrm{ml})$ for $0.5 \mathrm{~h}$, antiHER3 monoclonal therapeutic antibody (clone 3D4) $(5 \mu \mathrm{g} / \mathrm{ml})$ for $2.5 \mathrm{~h}$, and recombinant NRG1 $(100 \mathrm{ng} / \mathrm{ml})$ for $10 \mathrm{~min}$.

Trastuzumab (Herceptin) was purchased from Shanghai Roche Pharmaceuticals Co., Ltd. The anti-human HER3 monoclonal antibody (clone 3D4) was generously provided by Beijing Cotimes Biotech Co., Ltd. Recombinant NRG1 was purchased from Cell Signaling Technology. Primary antibodies to p-HER2/ErbB2 (Tyr1196) (1:1500), HER2/ErbB2 (1:1500), p-HER3/ErbB3 (Tyr1289) (1:1500), HER3/ErbB3 (1:1500), p-Akt (Ser473) (1:1500), Akt (pan) (1:1500), p-MAPK (Thr202/Tyr204) (1:1500), MAPK (1:1500) were purchased from Cell Signaling Technology. $\beta$-actin rabbit polyclonal antibody (1:1500) was purchased from Beijing Guan Xing Yu Sci-Tech Co., Ltd. Peroxidase-conjugated AffiniPure goat antirabbit IgG $(\mathrm{H}+\mathrm{L})$ (1:5000) was purchased from ZSGB-Bio.

SDS-PAGE and western blotting. After BCA quantitative operation, cell proteins were boiled at $99^{\circ} \mathrm{C}$ for $5 \mathrm{~min}$ in $5 \mathrm{X}$ buffer with protein phosphatase inhibitor (1:100) and then electrophoresed on a $10 \%$ SDS-PAGE gel. Target protein was transferred to a polyvinylidene fluoride membranes, and the membrane was blocked with skim milk (5\%) for $1 \mathrm{~h}$. After 2 washes with phosphate buffer solution (PBS), membranes were incubated with primary antibodies at $4^{\circ} \mathrm{C}$ overnight. After 3 washes with PBS, membranes were incubated with peroxidase-conjugated second antibodies for $1 \mathrm{~h}$. After 3 washes with PBS, membranes were colored using the Immobilon Western Chemiluminescent HRP Substrate (Millipore, Billerica, MA, USA).

RNA interference transfection in MDA-MB-453 cells. A small interfering RNA (siRNA) targeting human HER3 was purchased from Gene Pharma Co., Ltd. (Shanghai, China). siRNA transfection was performed in 6-well plates using Lipofectamine $^{\mathrm{TM}} 3000$ Reagent (Thermo Fisher Scientific, 

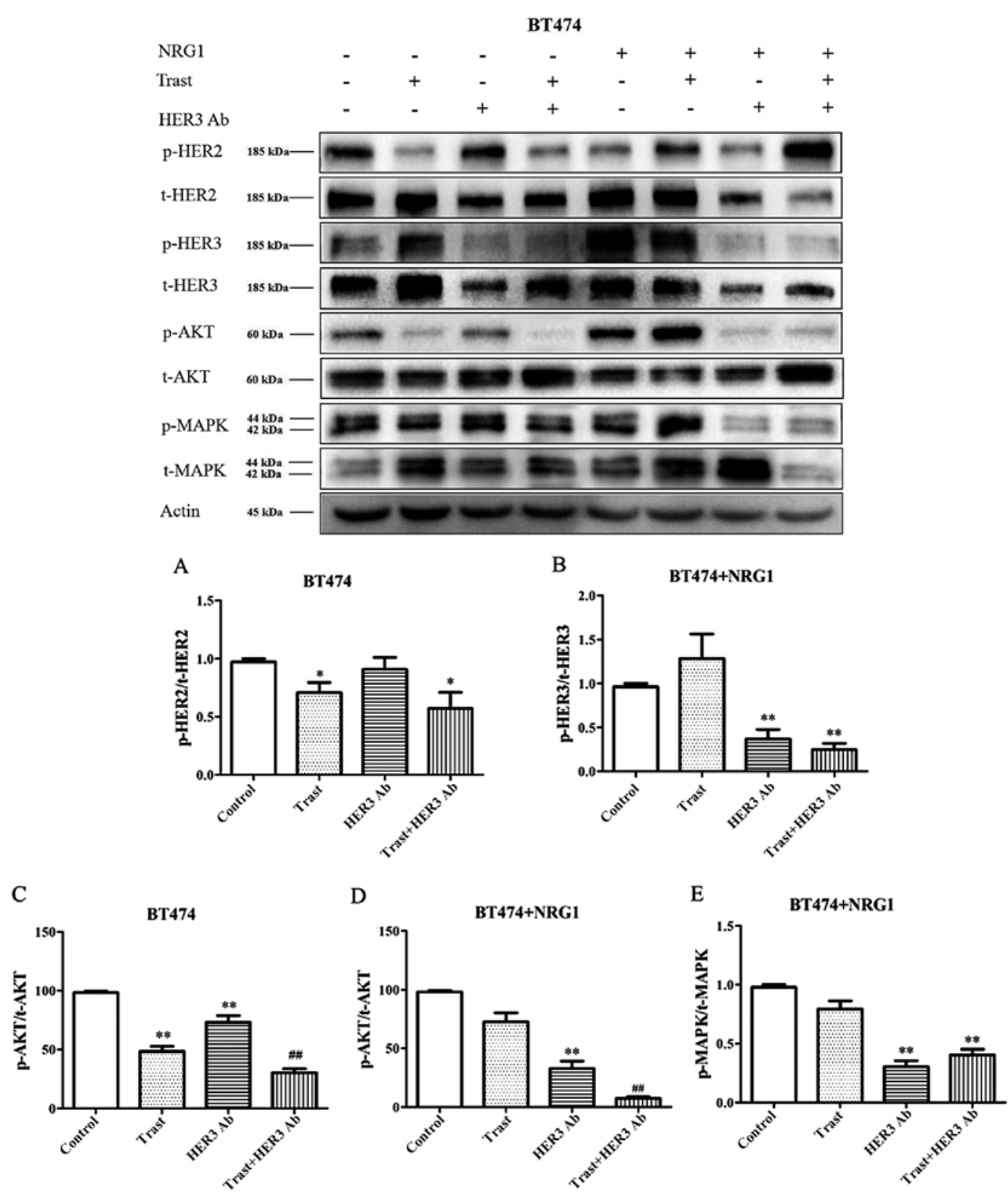

Figure 1. Activation of NRG1-dependent HER3 abolishes inhibitory effects of trastuzumab in BT474 cells. (A) p-HER2 was significantly downregulated by trastuzumab without NRG1, however, the downregulation effect disappeared after NRG1 activation. (B) p-HER3 was significantly downregulated by HER3 antibody after NRG1 stimulation. (C and D) The inhibitory effect of the HER3 antibody is superior to trastuzumab on p-Akt after NRG1 stimulation. (E) The expression of p-MAPK was only significantly downregulated by HER3 antibody after NRG1 stimulation. ${ }^{*} \mathrm{P}<0.05,{ }^{* *} \mathrm{P}<0.01$ compared to the control. ${ }^{\# \#} \mathrm{P}<0.01$ compared to trastuzumab or HER3 antibody. 'p-' represents 'phospho of'; 't-' represents 'total about of'.

Waltham, MA, USA) according to the manufacturer's protocols. Approximately 4000-5000 cells were seeded on each well on 6-well culture plate, and allowed to adhere overnight in supplemented medium. Lipofectamine 3000 Reagent (7.5 $\mu$ l) was diluted in Opti-MEM ${ }^{\mathrm{TM}}$ Medium $(125 \mu \mathrm{l})$, and mixed well. Dilute DNA in Opti-MEM and mix well to prepared DNA master mix. Mix diluted DNA and diluted Lipofectamine 3000 Reagent (1:1 ratio) in one tube and set to incubate for 10-15 $\mathrm{min}$ at room temperature. DNA-lipid complex was then added to cells, which were set to incubate cells for $48 \mathrm{~h}$ at $37^{\circ} \mathrm{C}$.

Apoptotic assay. Approximately $70 \%$ of the cell density per well were seeded on 6-well plates. The two types of cells, including the positive and negative controls, were incubated with different reagents for an appropriate time to induce apoptosis, followed by 2 washes with PBS, and then separated the cells from the well with $450 \mu \mathrm{l}$ of $0.25 \%$ trypsin-EDTA solution and re-suspended with $200 \mu \mathrm{l}$ of their respective culture medium. After that, $100 \mu \mathrm{l}$ of cells in suspension and $100 \mu \mathrm{l}$ of Muse $^{\mathrm{TM}}$ Annexin V and Dead Cell Reagent was added to each tube and incubated for $20 \mathrm{~min}$ at room temperature. Finally, the percentage of apoptotic cells was detected using the Muse Cell Analyzer (Muse 1.4 Analysis, Millipore).

Cell viability assay. Approximately 10000 cells per well were plated onto 96-well plates, and adhered at least $24 \mathrm{~h}$ in culture medium. The treatments were divided into four 


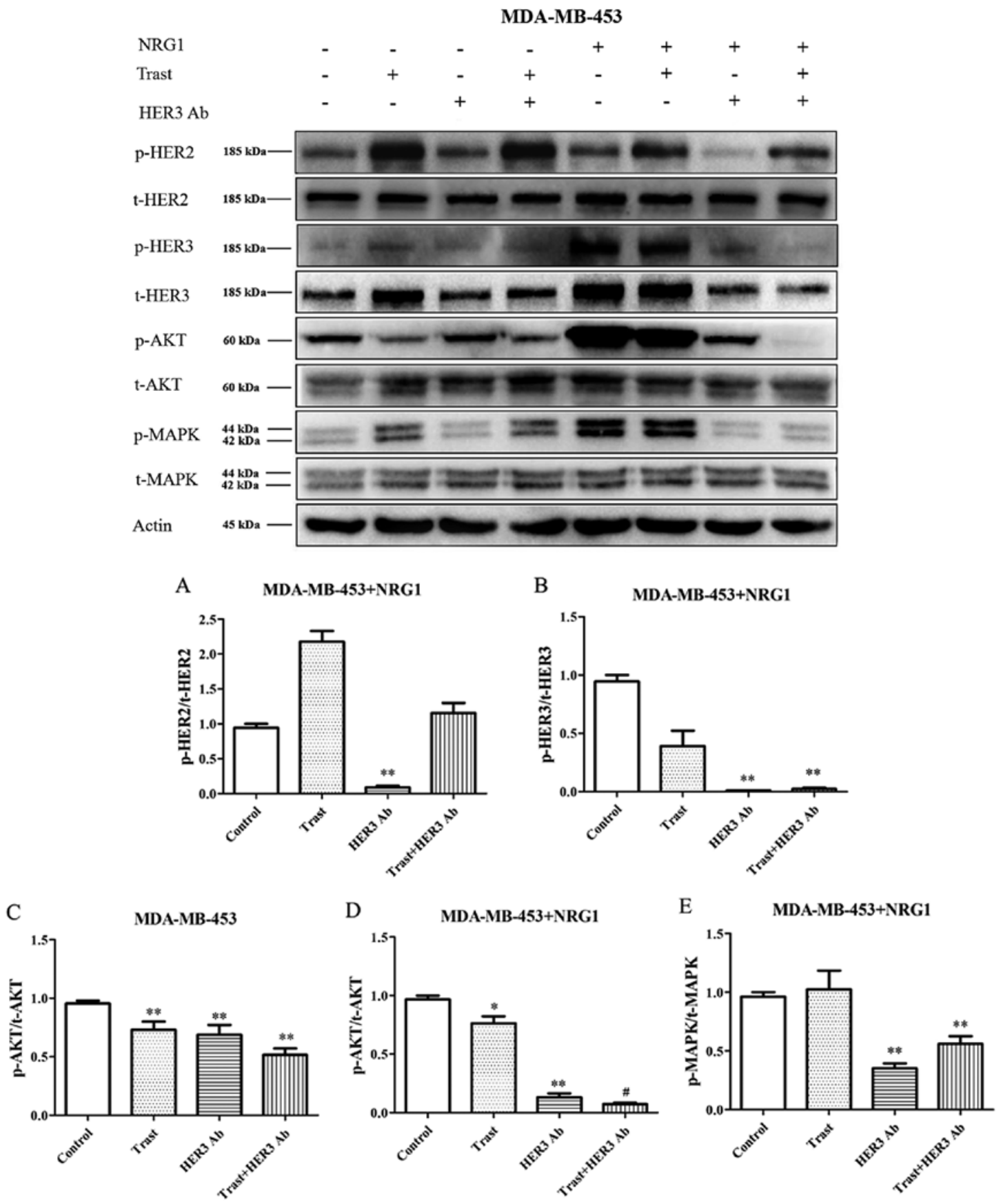

Figure 2. The NRG1-dependent HER3 antibody inhibits p-HER2/p-HER3/p-Akt (p-MAPK) pathways in MDA-MB-453 cells. (A and B) p-HER2 and p-HER3 were significantly downregulated by HER3 antibody after NRG1 stimulation. (C and D) HER3 antibody downregulated p-Akt without NRG1 stimulation, however, it became more effective with NRG1 stimulation. (E) The expression of p-MAPK was significantly downregulated by HER3 antibody after NRG1 stimulation. " $\mathrm{P}<0.05,{ }^{* *} \mathrm{P}<0.01$ compared to the control. " $\mathrm{P}<0.05$ compared to trastuzumab or HER3 antibody. 'p-' represents 'phospho of'; 't-' represents 'total about of'.

groups (control group, HER3 antibody alone group, trastuzumab alone group, and trastuzumab combined with HER3 antibody group) in a range of drug concentrations $(0.1,1,10$, 100 and $1000 \mathrm{nM}$ ), and then incubated for $48 \mathrm{~h}$ in the presence or absence of NRG1 (5 nM). The final live cell number was determined by thiazolyl blue tetrazolium bromide (MTT; Amresco LLC, Solon, OH, USA).

Statistical analysis. GraphPad Prism 5.01 (GraphPad Software Inc., USA) were used to perform the statistical analysis. Data obtained from western blotting were analyzed by AlphaView
SA 3.4.0 (Protein Simple, San Jose, CA, USA). The comparison between any two groups was determined by unpaired t-test or one-way ANOVA. A P-value of $<0.05$ was considered to indicate a statistically significant difference. Each experiment was repeated at least three times.

\section{Results}

Activation of NRG1-dependent HER3 abolished inhibitory effects of trastuzumab in BT474 cells. Without NRG1 stimulation, the expression of p-HER2 was significantly 

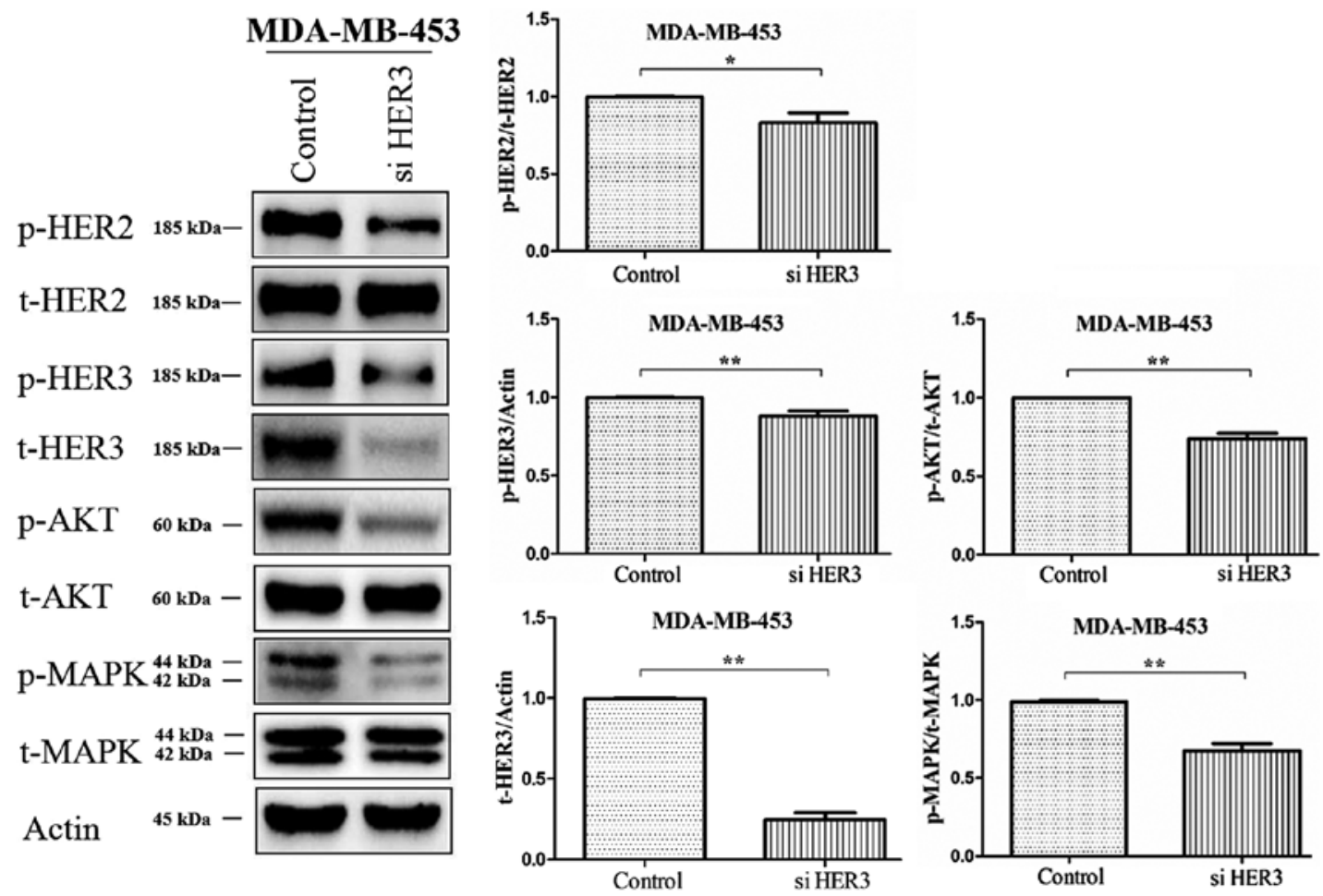

Figure 3. The interference effect of siHER3. After silencing HER3 gene, HER3 was obviously knocked down, the expression of p-HER2, p-HER3, p-Akt and p-MAPK was significantly downregulated compared to the control. ${ }^{*} \mathrm{P}<0.05,{ }^{* *} \mathrm{P}<0.01$.

downregulated by trastuzumab $(\mathrm{P}<0.05)$, whereas, the inhibitory effect of trastuzumab disappeared after NRG1 stimulation (Fig. 1). The HER3 antibody could not downregulate p-HER2 expression before NRG1 stimulation, but it showed a slight downregulation effect after NRG1 stimulation. The expression of p-HER3 was significantly increased after NRG1 stimulation (Fig. 1). The HER3 antibody had an inhibitory effect on p-HER3 before NRG1 stimulation, however, the downregulation role was significantly enhanced after NRG1 stimulation $(\mathrm{P}<0.01)$. The HER3 antibody combined with trastuzumab showed synergistic inhibitory effect on p-HER3 (Fig. 1).

After BT474 cells were stimulated with NRG1, the expression of p-Akt was upregulated. In the absence of NRG1 stimulation, the expression of p-Akt was significantly downregulated by trastuzumab and HER 3 antibody $(\mathrm{P}<0.01)$, but the inhibitory effect of trastuzumab was superior to that of the HER3 antibody. Whereas, with NRG1 stimulation, the inhibitory effect of trastuzumab decreased and the inhibitory effect of the HER3 antibody became more obvious $(\mathrm{P}<0.01)$. Combined application of the two antibodies showed synergistic effect (Fig. 1C and D). Without stimulation of NRG1, the expression of phospho mitogen-activated protein kinase (p-MAPK) was inhibited by trastuzumab in BT474 cells. The inhibitory effect of trastuzumab disappeared after NRG1 stimulation, however, the HER3 antibody showed significant downregulation effect $(\mathrm{P}<0.01)$ (Fig. 1E).

NRG1-depedent HER3 antibody inhibited p-HER2/pHER3/p-Akt(p-MAPK) pathways in MDA-MB-453 cells. The expression of p-HER3 and HER3 was significantly increased after NRG1 stimulation. The expression of p-HER2 and p-HER3 was not downregulated by trastuzumab without or with NRG1 stimulation (Fig. 2). After NRG1 stimulation, the expression of p-HER 2 and p-HER 3 were significantly downregulated by the HER3 antibody $(\mathrm{P}<0.01)$ (Fig. 2A and B).

In the absence of NRG1 stimulation, trastuzumab and HER3 antibody significantly downregulated the expression of p-Akt $(\mathrm{P}<0.01)$, and trastuzumab combined with the HER3 antibody seemed to have a synergistic trend (Fig. 2C). By adding NRG1 stimulation, the expression of p-Akt was significant increased, and the HER3 antibody significantly downregulated the raised p-Akt expression, moreover, the HER3 antibody combined with trastuzumab showed significant synergistic effect $(\mathrm{P}<0.05)$ (Fig. 2D). After NRG1 stimulation, trastuzumab did not downregulate p-MAPK expression, however, the HER3 antibody still significantly downregulated p-MAPK expression $(\mathrm{P}<0.01)$ (Fig. 2E).

The silencing role of siHER 3 in MDA-MB-453 cells. The expression of HER2, HER3, Akt and MAPK was detected by western blotting after silencing HER3 gene in MDA-MB-453 cells. The results showed that the expression of p-HER3 was significantly downregulated after HER3 gene silencing. The expression of p-HER2, p-Akt and p-MAPK was also downregulated compared to the control (Fig. 3).

NRG1-depedent HER3 antibody promotes early apoptosis. Trastuzumab significantly induced an increase in the proportion of early apoptosis in BT474 cells without NRG1 stimulation $(\mathrm{P}<0.05)$. After NRG1 stimulation, the role of trastuzumab significantly decreased. However, HER3 antibody significantly promoted early apoptosis of BT474 cells compared to the control $(\mathrm{P}<0.05)$. The combination therapy did not show synergistic effect (Fig. 4). 


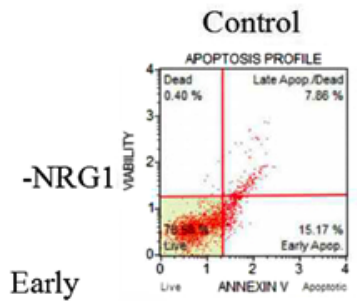

apoptotic:

$15.17 \%$

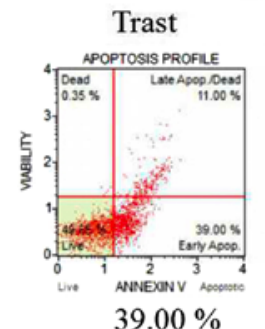

BT474

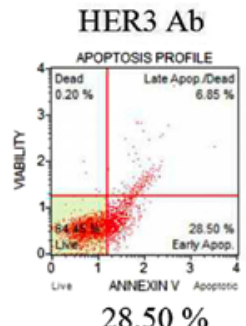

Trast+HER3 Ab

A
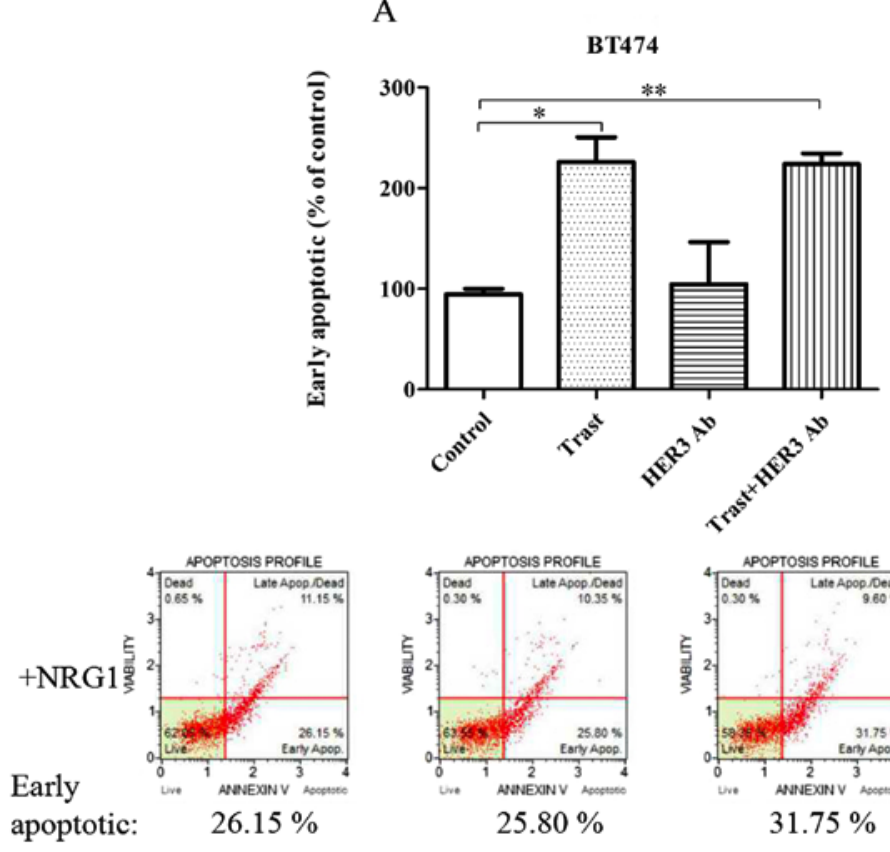

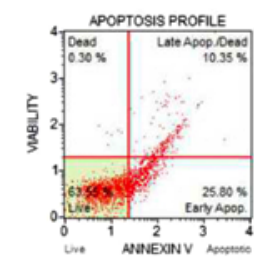

$25.80 \%$

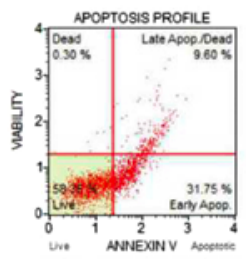

$31.75 \%$

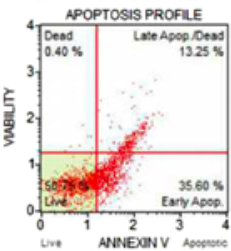

$35.60 \%$

B

BT474+NRG1

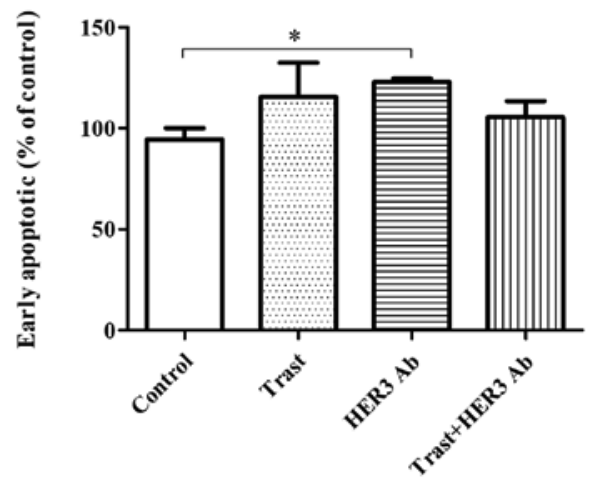

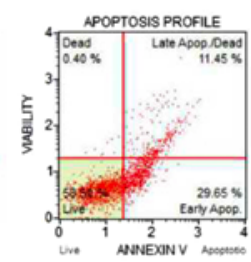

$29.65 \%$

Figure 4. The NRG1-dependent HER3 antibody promotes early apoptosis in BT474 cells. (A) Without NRG1 stimulation, trastuzumab significantly increased early apoptosis of BT474 cells when compared to the control (P<0.05). (B) After NRG1 stimulation, HER3 antibody significantly promoted early apoptosis compared to the control. ${ }^{*} \mathrm{P}<0.05,{ }^{* *} \mathrm{P}<0.01$ compared to the control.

In the absence of NRG1 stimulation, trastuzumab and HER3 antibody seemed to mildly induce an increase of early apoptosis in MDA-MB-453 cells. However, combined therapy showed significantly synergistic effect $(\mathrm{P}<0.05)$. After NRG1 stimulation, early apoptosis of MDA-MB-453 cells was not affected by trastuzumab, but it was significantly promoted by the HER3 antibody when compared to the control $(\mathrm{P}<0.01)$. The combined therapy seemed to have a synergistic trend (Fig. 5).

HER3 antibody combined with trastuzumab synergistically inhibits cell viability. In BT474 cells, with the increase of drug concentration, the inhibitory effect of trastuzumab on cell viability was gradually enhanced. While the effect of trastuzumab on MDA-MB-453 cells was weak and stable, even the drug concentration increased to $1000 \mathrm{nM}$. The inhibitory effects of trastuzumab on cell viability are different between the two cell lines. After NRG1 combined with trastuzumab, the cell viability was significantly increased in BT474 cells (Fig. 6A), however, this significant increase was not observed in MDA-MB-453 cells (Fig. 6D). With NRG1 stimulation, the combined therapy showed significantly inhibitory effect compared to trastuzumab treatment when the dosages were 

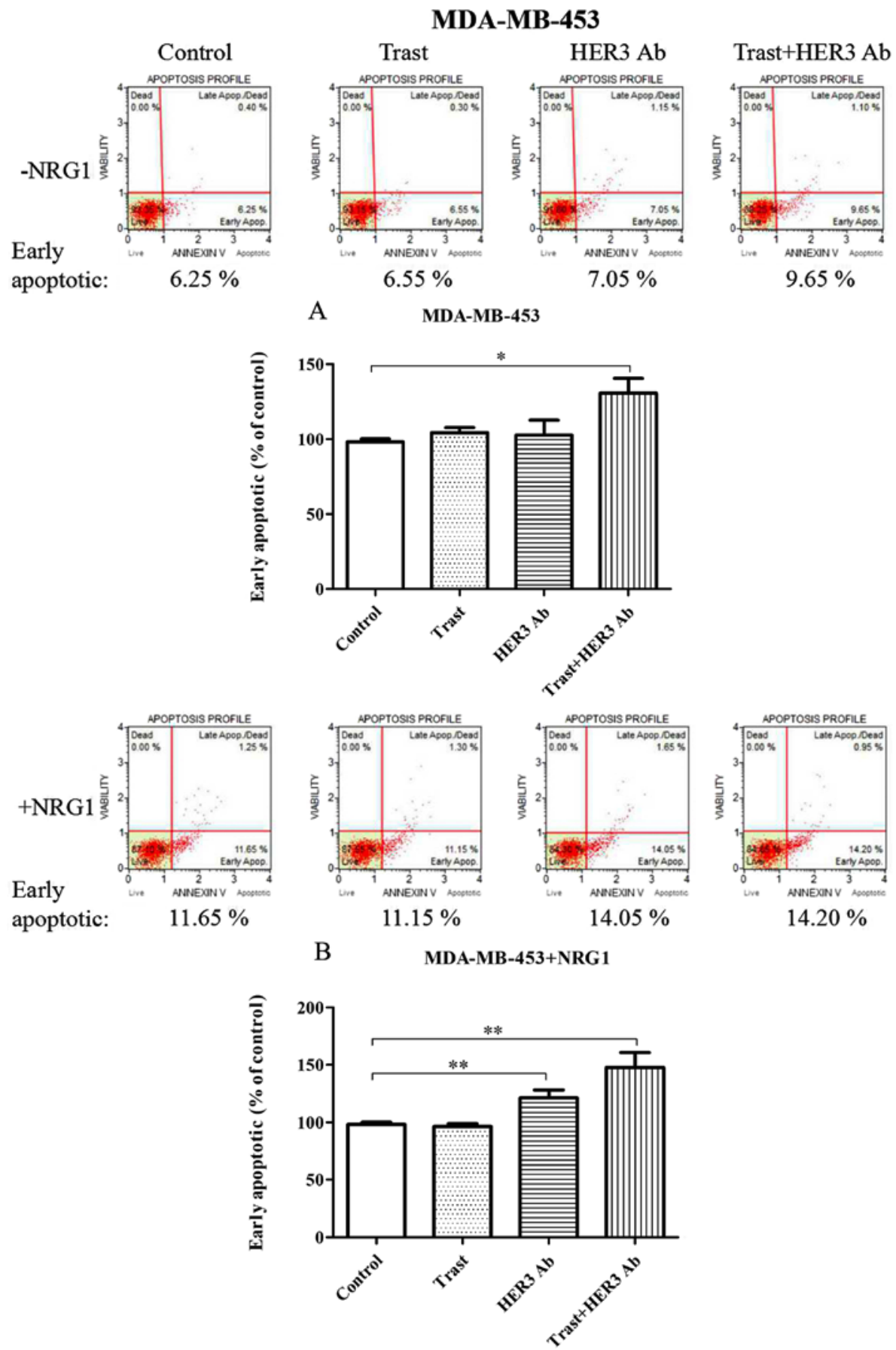

Figure 5. The NRG1-dependent HER3 antibody promotes the early apoptosis in MDA-MB-453 cells. (A) The combination therapy showed synergistic effect without NRG1 stimulation ( $\mathrm{P}<0.05)$. (B) After NRG1 stimulation, early apoptosis of MDA-MB-453 cells was significantly promoted by the HER3 antibody when compared to the control $(\mathrm{P}<0.01)$. The combined therapy seemed to have a synergistic trend. ${ }^{*} \mathrm{P}<0.05,{ }^{* * *} \mathrm{P}<0.01$ compared to the control.

higher than $1 \mathrm{nM}$ in BT474 and MDA-MB-453 cells (Fig. 6C and F). Synergistic inhibitory effect of combined therapy was observed at the concentration of $10 \mathrm{nM}$ in BT474 cells after NRG1 stimulation and at the concentration of $1 \mathrm{nM}$ and $10 \mathrm{nM}$ in MDA-MB-453 cells before NRG1 stimulation (Fig. 6C and E).

\section{Discussion}

HER3 was initially thought to be functionally passive and clinically insignificant. Currently, research has confirmed that HER3, as a functional activator, has the ability to activate recipient kinase (15). Moreover, HER3 is proved to be involved in tumorigenesis and progression, it may serve as a new therapeutic target (27). The research significance and clinical value of HER3 are becoming more and more important. A study by Lee-Hoeflich et al demonstrated that simultaneous downregulation of HER3 and HER2 was crucial in inhibiting proliferation of HER2-overexpressing breast cancer cells (17). Resistance to trastuzumab, which functions to block HER2, is a serious problem faced by clinicians. Therefore, the goal of our study was to analyze the role NRG1-depedent HER3 

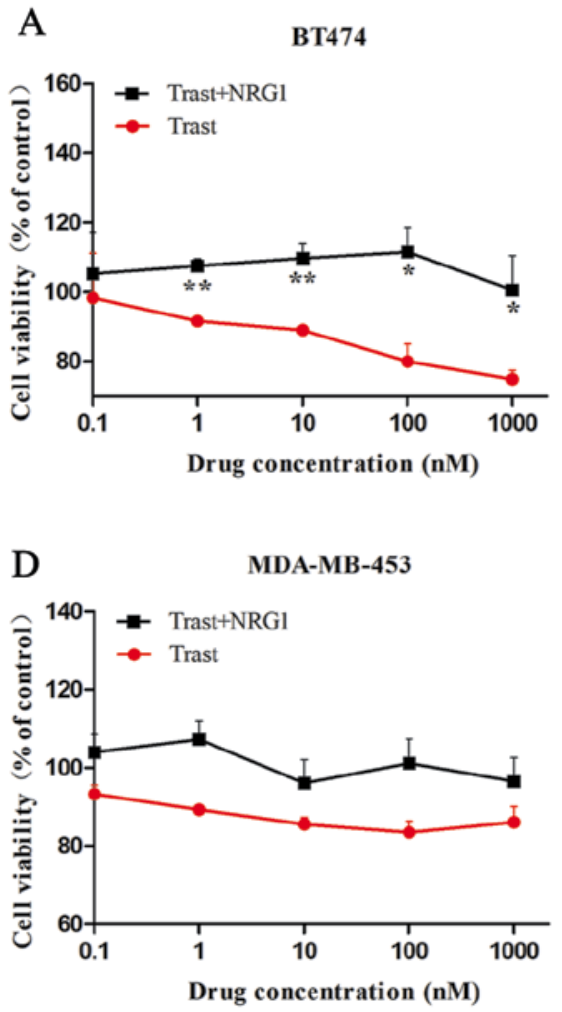

B

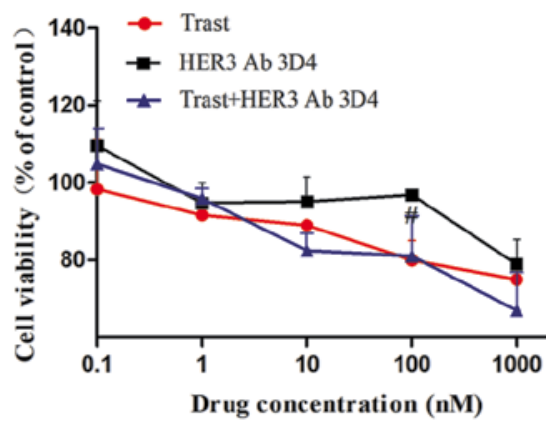

$\mathrm{E}$

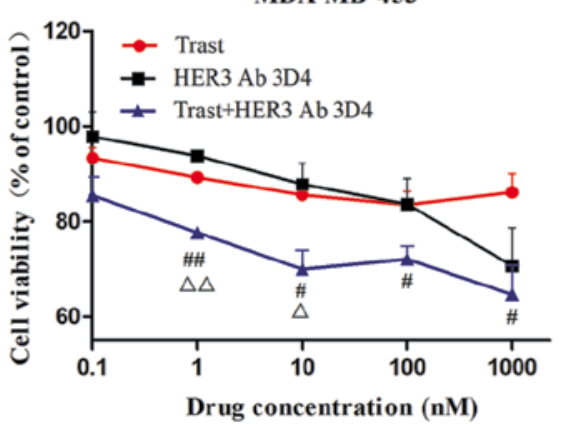

C

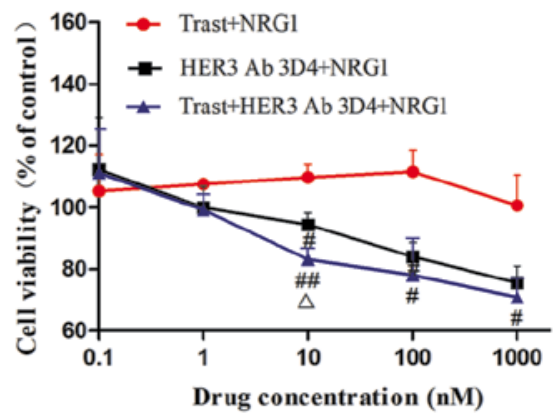

F

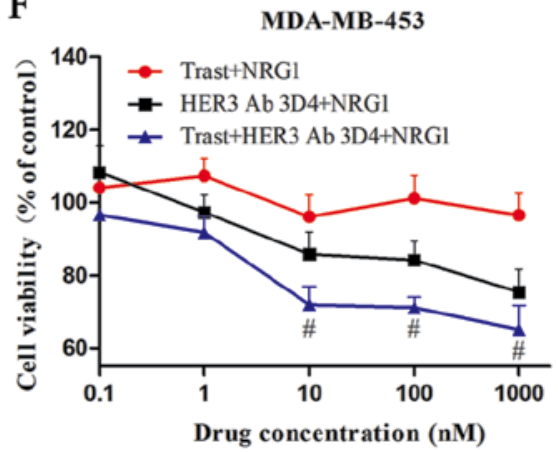

Figure 6. HER3 antibody combined with trastuzumab synergistically inhibits cell viability. (A and D) In BT474 cells, the inhibitory effect of trastuzumab on cell viability was gradually enhanced with the increase of drug concentration. However, the effect on MDA-MB-453 cells was weak and stable. After NRG1 combined with trastuzumab, the cell viability was significantly increased in BT474 cells, however, this significant increase was not observed in MDA-MB-453 cells. (C and F) With NRG1 stimulation, the combined therapy showed significantly inhibitory effect compared to trastuzumab treatment when the dosages were higher than $1 \mathrm{nM}$ in BT474 and MDA-MB-453 cells. (C and E) Synergistic inhibitory effect of combined therapy was observed at the concentration of $10 \mathrm{nM}$ in BT474 cells after NRG1 stimulation and at the concentration of 1 and $10 \mathrm{nM}$ in MDA-MB-453 cells before NRG1 stimulation. (A) ${ }^{*} \mathrm{P}<0.05,{ }^{* *} \mathrm{P}<0.01$ compared to trastuzumab. (B, C, E and F) ${ }^{\#} \mathrm{P}<0.05,{ }^{\# \#} \mathrm{P}<0.01$ compared to trastuzumab or trastuzumab+NRG1; ${ }^{\triangle} \mathrm{P}<0.05,{ }^{\triangle} \mathrm{P}<0.01$ compared to HER 3 antibody or HER3 antibody+NRG1.

activation has on inducing trastuzumab resistance and the inhibitory effect of HER3 monoclonal antibody on HER2overexpressing breast cancer cells.

In absence of NRG1 stimulation, trastuzumab significantly downregulated the expression of p-HER2 and induced an increase in early apoptosis in BT474 cells. After NRG1 stimulation, the inhibitory effects of trastuzumab disappeared. However, we observed that the expression of p-HER3 was increased after NRG1 stimulation. The addition of HER3 antibody significantly downregulated the expression of HER3 and induced an increase in early apoptosis. The data suggest that the p-HER3 upregulation, stimulated by NRG1, may counteract the p-HER2 downregulation effect caused by trastuzumab, potentially leading to trastuzumab resistance. A study by Garrett et al showed that trastuzumab and lapatinib double blockade of HER2 caused a transcriptional and posttranslational upregulation of HER3, partially offsetting the targeted inhibition efficacy of HER2-directed therapies (28). Our result coincides with that of Göstring et al, who concluded that the activation loop of NRG1/HER3 played a vital role in the proliferation process of HER2-overexpressing breast cancer cells, independently of HER2 (22). Trastuzumab did not downregulate p-HER2 or p-HER3 and had no effect on apoptosis before or after the addition of NRG1 to MDA-MB453 cells, confirming that MDA-MB-453 cells were indeed resistant to trastuzumab. After NRG1 stimulation, the HER3 antibody significantly downregulated p-HER2 and p-HER3 and significantly promoted apoptosis in MDA-MB-453 cells, indicating that the inhibition of NRG1/HER2/HER3 led to the apoptosis of MDA-MB-453 cells. Therefore, the activation of NRG1/HER3 may be one of the key factors causing primary resistance to trastuzumab in MDA-MB-453 cells. A study by Wu et al showed that antisense oligonucleotide (EZN-3920) could sustain an anti-proliferation effect in trastuzumabresistant cells by effectively downregulating the expression of HER3 (29). A study by Ebbing et al showed that the metalloproteinase ADAM10 activated HER3 and downstream signaling by releasing NRG1 from the cell membrane and induced resistance to trastuzumab (30). These studies suggest that NRG1/HER3 was closely related to trastuzumab resistance, and the inhibition of HER3 might reverse trastuzumab resistance. Moreover, after the silencing of HER3 gene, the expression of p-HER2, p-HER3 and the downstream proteins p-Akt and p-MAPK were significantly downregulated, consistent with the inhibitory effect of HER3 antibody.

For HER3 antibody (3D4), apoptotic-promoting effect was not present before NRG1 stimulation, but it was observed after NRG1 stimulation in MDA-MB-453 cells, indicating that HER3 antibody might reverse NRG1 initiated primary resistance to trastuzumab. A study by Leung et al showed that lapatinib, a HER1/HER2 small molecule tyrosine kinase inhibitor, could not continuously inhibit the 
signaling pathway of HER dimers. The elevation of NRG1 was observed in resistance process, whereas the addition of the HER3 antibody SPG1 effectively reversed the resistance to lapatinib (31), which was almost in line with our findings. A study by Xia et al showed that NRG1-positive expression was an independent negative predictive factor for HER2-overexpressing breast cancer, indirectly hinting that NRG1-dependent HER3 activation might promote the progression of breast cancer (32).

The expression of p-Akt and p-MAPK was downregulated by the HER3 antibody in BT474 and MDA-MB-453 cells after NRG1 stimulation. These results suggest that NRG1-dependent HER3 inducing the primary resistance to trastuzumab was mediated by Akt and MAPK-related signaling pathways. A study by Dey et al showed that simultaneous inhibition of the HER 2 and PI3K/Akt/mTOR signaling pathways was more effective than the single inhibition of HER2 (33). A combination of the new Akt inhibitor AZD5363 and the EGFR/HER2/ HER3 inhibitor AZD8931 synergistically inhibited the proliferation of HER2-overexpressing breast cancer cells $(34,35)$, and $\mathrm{S} 100 \mathrm{P}$ induced trastuzumab resistance by activating the RAS/MEK/MAPK signaling pathway (36). These findings were consistent with our results that the HER3 antibody promoted apoptosis in MDA-MB-453 cells by downregulating Akt and MAPK phosphorylation. Therefore, HER3 antibody reversed NRG1-induced trastuzumab primary resistance by interfering with the phosphorylation of HER3, blocking the formation of heterodimers, and downregulating p-Akt and p-MAPK in the downstream signaling pathway.

HER3 targeting therapy is rapidly developing. Sensitivity of colorectal cancer DiFi cells to cetuximab was restored by Patritumab, a HER3 monoclonal antibody, which functions by inhibiting the activity of NRG1/HER3 (37). HER3 monoclonal antibody LMAb3 fully inhibited proliferation of trastuzumabresistant SKOV3-T cells by downregulating the activity of HER3 and its related signaling proteins (38). Wang et al reported that HER3 monoclonal antibody and trastuzumab have synergistically inhibitory effect in HER2-positive gastric cancer cells (39). A study by Canonici et al showed that the irreversible panHER inhibitor Neratinib reversed trastuzumab-resistance (40). In our study, we found synergistic effects between trastuzumab and HER3 monoclonal antibody (3D4) in the inhibition of cell viability in both BT474 and MDA-MB-453 cells after NRG1 stimulation.

In conclusion, NRG1/HER3 activation is one of the key factors inducing primary resistance to trastuzumab in HER2overexpressing breast cancer cells. The HER3 antibody may reverse trastuzumab primary resistance by significantly inhibiting the activation of NRG1-dependent HER3. Because of the diversity and complexity of the HER family and the signal transduction pathways, the combined targeted drugs or multi-targeted drugs treatment may become the trend in the future. Trastuzumab combined with HER3 monoclonal antibody may be a treatment choice for patients with primary resistance to trastuzumab.

\section{Acknowledgements}

We thank Professor Wang Ping and Professor Zhang Dong for experimental guidance. Springer Nature Author Services helped to prepare our manuscript. This study was funded by the National Natural Science Foundation of China (no. 81301912, to Q.L.), the Beijing Municipal Health System High-level Health Person Foundation Project (no. 2014-3-005, to Q.L.), and the Beijing Municipal Science and Technology Commission (Capital Features, Z161100000516083, awarded to Q.L.).

\section{References}

1. Slamon DJ, Godolphin W, Jones LA, Holt JA, Wong SG, Keith DE, Levin WJ, Stuart SG, Udove J, Ullrich A, et al: Studies of the HER-2/neu proto-oncogene in human breast and ovarian cancer. Science 244: 707-712, 1989.

2. Schulz R, Streller F, Scheel AH, Rüschoff J, Reinert MC Dobbelstein M, Marchenko ND and Moll UM: HER2/ErbB2 activates HSF1 and thereby controls HSP90 clients including MIF in HER2-overexpressing breast cancer. Cell Death Dis 5: e980, 2014.

3. Slamon DJ, Clark GM, Wong SG, Levin WJ, Ullrich A and McGuire WL: Human breast cancer: Correlation of relapse and survival with amplification of the HER-2/neu oncogene. Science 235: 177-182, 1987.

4. Nahta R: Molecular mechanisms of trastuzumab-based treatment in HER2-overexpressing breast cancer. ISRN Oncol 2012: 428062, 2012.

5. Gelmon KA, Boyle FM, Kaufman B, Huntsman DG, Manikhas A Di Leo A, Martin M, Schwartzberg LS, Lemieux J, Aparicio S, et al: Lapatinib or trastuzumab plus taxane therapy for human epidermal growth factor receptor 2-positive advanced breast cancer: Final results of NCIC CTG MA.31. J Clin Oncol 33: 1574-1583, 2015.

6. Valero V, Forbes J, Pegram MD, Pienkowski T, Eiermann W, von Minckwitz G, Roche H, Martin M, Crown J, Mackey JR, et al: Multicenter phase III randomized trial comparing docetaxel and trastuzumab with docetaxel, carboplatin, and trastuzumab as first-line chemotherapy for patients with HER2-gene-amplified metastatic breast cancer (BCIRG 007 study): Two highly active therapeutic regimens. J Clin Oncol 29: 149-156, 2011.

7. Ritter CA, Perez-Torres M, Rinehart C, Guix M, Dugger T, Engelman JA and Arteaga CL: Human breast cancer cells selected for resistance to trastuzumab in vivo overexpress epidermal growth factor receptor and ErbB ligands and remain dependent on the ErbB receptor network. Clin Cancer Res 13: 4909-4919, 2007.

8. Madrid-Paredes A, Cañadas-Garre M, Sánchez-Pozo A and Calleja-Hernández MA: Non-HER2 signaling pathways activated in resistance to anti-HER2 therapy in breast cancer. Breast Cancer Res Treat 153: 493-505, 2015.

9. Scaltriti M, Rojo F, Ocaña A, Anido J, Guzman M, Cortes J, D Cosimo S, Matias-Guiu X, Ramon y Cajal S, Arribas J, et al: Expression of p95HER2, a truncated form of the HER2 receptor, and response to anti-HER 2 therapies in breast cancer. J Natl Cancer Inst 99: 628-638, 2007.

10. Ozkavruk Eliyatkin N, Aktas S, Ozgur H, Ercetin P and Kupelioglu A: The role of p95HER2 in trastuzumab resistance in breast cancer. J BUON 21: 382-389, 2016.

11. Narayan M, Wilken JA, Harris LN, Baron AT, Kimbler KD and Maihle NJ: Trastuzumab-induced HER reprogramming in 'resistant' breast carcinoma cells. Cancer Res 69: 2191-2194, 2009.

12. Deguchi Y, Okabe H, Oshima N, Hisamori S, Minamiguchi S Muto $\mathrm{M}$ and Sakai Y: PTEN loss is associated with a poor response to trastuzumab in HER2-overexpressing gastroesophageal adenocarcinoma. Gastric Cancer 20: 416-427, 2017.

13. Luque-Cabal M, García-Teijido P, Fernández-Pérez Y, SánchezLorenzo L and Palacio-Vázquez I: Mechanisms behind the resistance to Trastuzumab in HER2-amplified breast cancer and strategies to overcome it. Clin Med Insights Oncol 10 (Suppl 1): 21-30, 2016.

14. Liu J, Pan C, Guo L, Wu M, Guo J, Peng S, Wu Q and Zuo Q: A new mechanism of trastuzumab resistance in gastric cancer: MACC1 promotes the Warburg effect via activation of the PI3K/AKT signaling pathway. J Hematol Oncol 9: 76, 2016.

15. Jura N, Shan Y, Cao X, Shaw DE and Kuriyan J: Structural analysis of the catalytically inactive kinase domain of the human EGF receptor 3. Proc Natl Acad Sci USA 106: 21608-21613, 2009. 
16. Shi F, Telesco SE, Liu Y, Radhakrishnan R and Lemmon MA: ErbB3/HER3 intracellular domain is competent to bind ATP and catalyze autophosphorylation. Proc Natl Acad Sci USA 107: 7692-7697, 2010

17. Lee-Hoeflich ST, Crocker L, Yao E, Pham T, Munroe X, Hoeflich KP, Sliwkowski MX and Stern HM: A central role for HER3 in HER2-amplified breast cancer: Implications for targeted therapy. Cancer Res 68: 5878-5887, 2008.

18. Jeong H, Kim J, Lee Y, Seo JH, Hong SR and Kim A: Neuregulin-1 induces cancer stem cell characteristics in breast cancer cell lines. Oncol Rep 32: 1218-1224, 2014.

19. Sheng Q, Liu X, Fleming E, Yuan K, Piao H, Chen J, Moustafa Z, Thomas RK, Greulich H, Schinzel A, et al: An activated ErbB3/ NRG1 autocrine loop supports in vivo proliferation in ovarian cancer cells. Cancer Cell 17: 298-310, 2010.

20. De Boeck A, Pauwels P, Hensen K, Rummens JL, Westbroek W, Hendrix A, Maynard D, Denys H, Lambein K, Braems G, et al: Bone marrow-derived mesenchymal stem cells promote colorectal cancer progression through paracrine neuregulin 1/HER3 signalling. Gut 62: 550-560, 2013.

21. Kim S, Han J, Shin I, Kil WH, Lee JE and Nam SJ: A functional comparison between the HER2(high)/HER3 and the HER2(low)/HER3 dimers on heregulin- $\beta 1$-induced MMP-1 and MMP-9 expression in breast cancer cells. Exp Mol Med 44: 473-482, 2012

22. Göstring L, Malm M, Höidén-Guthenberg I, Frejd FY, Ståhl S, Löfblom J and Gedda L: Cellular effects of HER3-specific affibody molecules. PLoS One 7: e40023, 2012.

23. Ni M, Chen Y, Lim E, Wimberly H, Bailey ST, Imai Y, Rimm DL, Liu XS and Brown M: Targeting androgen receptor in estrogen receptor-negative breast cancer. Cancer Cell 20: 119-131, 2011.

24. Shim JS, Rao R, Beebe K, Neckers L, Han I, Nahta R and Liu JO: Selective inhibition of HER2-positive breast cancer cells by the HIV protease inhibitor nelfinavir. J Natl Cancer Inst 104: 1576-1590, 2012.

25. Barok M, Tanner M, Köninki K and Isola J: Trastuzumab-DM1 causes tumour growth inhibition by mitotic catastrophe in trastuzumab-resistant breast cancer cells in vivo. Breast Cancer Res 13: R46, 2011

26. Walsh AJ, Cook RS, Sanders ME, Aurisicchio L, Ciliberto G Arteaga CL and Skala MC: Quantitative optical imaging of primary tumor organoid metabolism predicts drug response in breast cancer. Cancer Res 74: 5184-5194, 2014.

27. Li Q, Yuan Z and Cao B: The function of human epidermal growth factor receptor-3 and its role in tumors (Review). Oncol Rep 30: 2563-2570, 2013.

28. Garrett JT, Sutton CR, Kuba MG, Cook RS and Arteaga CL: Dual blockade of HER2 in HER2-overexpressing tumor cells does not completely eliminate HER3 function. Clin Cancer Res 19: 610-619, 2013

29. Wu Y, Zhang Y, Wang M, Li Q, Qu Z, Shi V, Kraft P, Kim S, Gao Y, Pak J, et al: Downregulation of HER 3 by a novel antisense oligonucleotide, EZN-3920, improves the antitumor activity of EGFR and HER2 tyrosine kinase inhibitors in animal models. Mol Cancer Ther 12: 427-437, 2013
30. Ebbing EA,Medema JP,Damhofer H,Meijer SL, Krishnadath KK, van Berge Henegouwen MI, Bijlsma MF and van Laarhoven HW: ADAM10-mediated release of heregulin confers resistance to trastuzumab by activating HER3. Oncotarget 7: 10243-10254, 2016.

31. Leung WY, Roxanis I, Sheldon H, Buffa FM, Li JL, Harris AL and Kong A: Combining lapatinib and pertuzumab to overcome lapatinib resistance due to NRG1-mediated signalling in HER2amplified breast cancer. Oncotarget 6: 5678-5694, 2015.

32. Xia W, Petricoin EF III, Zhao S, Liu L, Osada T, Cheng Q, Wulfkuhle JD, Gwin WR, Yang X, Gallagher RI, et al: An heregulin-EGFR-HER3 autocrine signaling axis can mediate acquired lapatinib resistance in HER $2^{+}$breast cancer models. Breast Cancer Res 15: R85, 2013

33. Dey N, Sun Y, Carlson JH, Wu H, Lin X, Leyland-Jones B and De P: Anti-tumor efficacy of BEZ235 is complemented by its anti-angiogenic effects via downregulation of PI3K-mTORHIF1alpha signaling in HER2-defined breast cancers. Am J Cancer Res 6: 714-746, 2016.

34. Crafter C, Vincent JP, Tang E, Dudley P, James NH, Klinowska T and Davies BR: Combining AZD8931, a novel EGFR/HER2/ HER3 signalling inhibitor, with AZD5363 limits AKT inhibitor induced feedback and enhances antitumour efficacy in HER2amplified breast cancer models. Int J Oncol 47: 446-454, 2015.

35. O'Brien NA, McDonald K, Tong L, von Euw E, Kalous O, Conklin D, Hurvitz SA, di Tomaso E, Schnell C, Linnartz R, et al: Targeting PI3K/mTOR overcomes resistance to HER2targeted therapy independent of feedback activation of AKT. Clin Cancer Res 20: 3507-3520, 2014.

36. Merry CR, McMahon S, Forrest ME, Bartels CF, Saiakhova A, Bartel CA, Scacheri PC, Thompson CL, Jackson MW, Harris LN, et al: Transcriptome-wide identification of mRNAs and lincRNAs associated with trastuzumab-resistance in HER2positive breast cancer. Oncotarget 7: 53230-53244, 2016.

37. Kawakami H, Okamoto I, Yonesaka K, Okamoto K, Shibata K, Shinkai Y, Sakamoto H, Kitano M, Tamura T, Nishio K, et al: The anti-HER3 antibody patritumab abrogates cetuximab resistance mediated by heregulin in colorectal cancer cells. Oncotarget 5: 11847-11856, 2014

38. Li X, Duan Y, Qiao C, Zhou T, Yu M, Geng J, Feng J, Shen B, LV M and Li Y: Anti-HER3 monoclonal antibody inhibits acquired trastuzumab-resistant gynecologic cancers. Technol Cancer Res Treat 15: 573-582, 2016.

39. Wang Q, Zhang X, Shen E, Gao J, Cao F, Wang X, Li Y, Tian T, Wang J, Chen Z, et al: The anti-HER3 antibody in combination with trastuzumab exerts synergistic antitumor activity in HER2positive gastric cancer. Cancer Lett 380: 20-30, 2016.

40. Canonici A, Gijsen M, Mullooly M, Bennett R, Bouguern N, Pedersen K, O'Brien NA, Roxanis I, Li JL, Bridge E, et al: Neratinib overcomes trastuzumab resistance in HER2 amplified breast cancer. Oncotarget 4: 1592-1605, 2013. 